\title{
Elements of effective practice in patient-centred laboratory medicine
}

\author{
Chit Tam ${ }^{1}$, William Chi-Shing Cho ${ }^{2 *}$ and Tzi Bun $\mathrm{Ng}^{1 *}$ \\ ${ }^{1}$ School of Biomedical Sciences, Faculty of Medicine, The Chinese University of Hong Kong, China \\ ${ }^{2}$ Department of Clinical Oncology, Queen Elizabeth Hospital, Hong Kong, Hong Kong, China
}

\begin{abstract}
Laboratory medicine is an evidence-based medicine which involves a set of medical diagnosis conducted on tissues, fluids, or other specimens to assist clinical staff and medical doctors in disease diagnosis and patients treatment. Chemical, cytological, haematological, microbiological, histological, and molecularly techniques are employed in the process of acquiring the diagnostic results. Quality assurance of the medical laboratory is regulated and accredited by international standardisation organisations. The whole process of patient-centred laboratory medicine could be described into five stages: screening of diseases, risk identification and stratification, clinical diagnosis, selection of medical treatment by physicians and continuous monitoring of drug efficacy and side effects. It was reported that the pre-analytical process is the major laboratory errors and thus elimination of these errors is critical for maintaining patient-centred laboratory medicine. Advanced development of molecular medicine and automation of clinical system requires development of laboratory medicine while systematic quality management should be maintained for good quality of medical practice. Foreseeably further laboratory medicine may contribute to targeted therapy and personalised management.
\end{abstract}

\section{Introduction}

\section{Change of laboratory medicine: From additional auxiliary} for medical plan to first-line diagnosis

Laboratory medicine is an evidence-based medicine which is a set of medical diagnosis conducted on tissues, fluids, or other specimens to assist clinical staff and medical doctors in disease diagnosis and patients, treatment [1,2]. Chemical, cytological, haematological, microbiological, histological, and molecularly techniques are employed in the process of acquiring the diagnostic results. The medical laboratory has developed for over a century. It originally served in implementing an auxiliary procedure for disease identification. In the early $20^{\text {th }}$ century, laboratory testing was merely engaged in conducting some basic chemical and physiological analyses like glycolytic analysis, blood gas, and microscopic analyses like counts of blood cells and identification of bacterial or fungal species causing an infection. Aided by the industrial and informatics development as well as the advanced research studies in genomics, proteomics, metabolomics and the corresponding biomedical relationship, clinical diagnosis is becoming more and more important. Currently medical planning is determined not only by clinical analysis from physicians but also relies on the medical report from laboratory medicine. In today's hospital systems, Over $70 \%$ of the decisions of medical treatments rely on diagnostic results from the medical laboratory [3] which implies that laboratory medicine has become the first-line health monitoring to the public. Therefore, quality control, assurance and management of laboratory medicine are essential to ensure the high quality of public health.

\section{Patient-centred laboratory medicine and standardisation}

Accuracy and precision of the test results are essential criteria for laboratory medicine, hence, systematic quality assurance is the cornerstone of clinical laboratory processing. There are two associated systems for medical laboratory accreditation: International standard system for medical laboratory, ISO15189:2012 offered by International Organization for Standardization $[4,5]$ which provides international standards in medical laboratory and, a US system, accreditation of laboratory through College of American Pathologists (CAP accreditation) which is an accreditation programme based on ISO15189[6].

\section{Building blocks of patient-centred laboratory medicine and improvement}

\section{Essential elements in patient-centred laboratory medicine}

The key elements in patient-centred laboratory medicine can be described in 5 processes, including disease screening, risk identification and stratification, diagnostic analysis, medical treatment selection and follow-up monitoring. During the screening, physicians may make a primary study of the cause of the disease by clinical investigation and examination during the consultation and identify the risk factors of the patients. Based on the proposed risk factors, they will request the patients to provide specimens for disease screening and diagnosis. Blood specimens are usually collected by a phlebotomist and while faecal and urine-related specimens are usually collected by point-ofcare (POC) kits or special collection bottles provided by patients. The

Correspondence to: Tzi Bun Ng, Faculty of Medicine, The Chinese University of Hong Kong, China, E-mail: tzibunng@cuhk.edu.hk

William Chi-Shing Cho, Department of Clinical Oncology, Queen Elizabeth Hospital, China, E-mail: chocs@ha.org.hk

Key words: patient-centred medicine, clinical laboratory management, automation

Received: November 29, 2017; Accepted: December 23, 2017; Published: December 27, 2017 
specimens will be stored at the appropriate condition and delivered to the laboratory (i.e. pre-analytical process). Hence, during screening and risk stratification, the key elements are investigational validity and accuracy, collection method, delivery condition, temporary storage time and, disease and risk classification.

Diagnostic testing (analytical and pre-analytical process) contains the best quality control because medical standardisation and quality management system like ISO15189 are well established. There is a list of analytical variables that need to be monitored including sample labelling and registration, equipment and device performance, chemical validity, automated system performance, sample quality control, management of documentation for protocols, test reports, clinical reports, audit/quality assurance certificates and maintenance records, staff training and performance, environmental quality control etc. To rule out the uncertainty, planning for a regular internal audit for quality assurance (e.g. once every 6 months) and an annual external audit is suggested.

The most suitable treatment plan will be given to the patients after the final decision from medical doctors which is based on the results of the clinical laboratory report as well as the actual health condition of the patients. However, the control of treatment selection cannot be well established because it relies on the clinical experience of the medical doctors. Therefore, physicians should have a continuous study of medical diagnosis and therapeutics. A continuous monitoring will also be carried out on the patients in order to confirm the efficacy of the drugs, health condition of the patients and early detection of the side effects. Any abnormal conditions found should be reported to the corresponding medical doctor who can then adjust the treatment plan immediately. Monitoring can be well managed by good recording and documentation practice. Automation and computing system for medical record may enhance the quality control in this process (Figure 1) [2,7].

Horvath and Pewsner suggested that regular systematic review on the quality management in medical laboratory is important. They introduced some key points of a systematic review protocol for laboratory medicine assessment including clinical study design, screening and selection of patients, validity of reference test materials, interpretation and analysis of the results [8].

To support effective practice in patient-centred laboratory medicine, the above key elements are critical and essential while they are not the end of the list. By the innovation of clinical diagnosis, further improvements in laboratory practice are necessarily important. In the following section improvement of patient-centred laboratory medicine in terms of error control, staff training, and innovative adaptation and technical transfer will be discussed.

\section{Root causes of clinical laboratory test failures and management}

In a clinical laboratory practice, for example, a hospital clinical laboratory, there are several points for generation of inadequate testing results including sample collection error (pre-analytical), testing error (analytical error) and data interpretation error (post-analytical error) (Figure 2) [3]. The major testing errors in the past could be about the human mediated events such as wrong labelling and mismatch of samples, addition of an incorrect volume of solution, using invalid reagents, and making errors in calculations etc. By the development of medical machine and system, machine-driven or system-driven errors will also be considered as one of the testing errors. However, nowadays testing errors and data interpretation errors are seldom found in the clinical laboratory in a hospital since the public hospital system has developed a fully automated system for clinical tests [3]. The automated system contains three key parts: an automated sample registration system, automated all-in-one diagnostic testing system and computer based analysis. Each part is well-established and organised with stagedependent examination or key point monitoring. As a result, analytical and post-analytical errors are under control.

On the other hand, pre-analytical errors are the critical issues, especially during specimen collection. For example, haemolysis due to temperature variation or induction of disinfectant and inadequate quantity during phlebotomy, high temperature used for temporary storage of stool specimens and biopsies, wrong collection procedure or even wrong labelling of specimens usually reduce the quality of the specimens. Since this is the source of the origin, low quality of specimens may generate invalid test results and affect the medical decision for patients. Therefore, control of pre-analytical procedures is definitely essential and crucial. To improve the quality and validity of laboratory medicine, regular examination and root cause analysis for the critical failure at pre-testing stage by setting up appropriateness of test requests and screening, patient identification, request and order management, sample collection, identification and transportation and acceptance/rejection criteria in accordance with the international standard ISO15189:2012 [9].

Meanwhile, there is a trend of point-of-care kit application in the public health system. It is a good idea that patients regularly examine their health condition outside hospitals in an easy and convenient way. However, there is always a big drawback is that the data collection is controlled by the patients themselves which may also become a source of discrepancy. Some of the tests like glycaemic test, a test for basal urine chemistry or faecal microbial composition analysis, strictly rely on the quality of the original source. Since the POC samples come from outpatients, there may be a potential variation against the true value for the following reasons: a patient may not follow the regulations to adjust his or her diet (like starvation), misuse of the POC kit, environmental variations to the POC kit and there is a small percentage of people who may even try to "manipulate" their sample source for a better result. Hence, POC kits may bring us an easier way for health monitoring and screening while control of the sample quality is still a challenging task for laboratory medicine. It is believed that proper education for homebased medical tests and training of first-line staff for sample collection is critical for pre-analytical quality management. Performance tests for the kit can be given to healthcare staff or even the patients if necessary.

\section{Ideas for future patient-centred laboratory medicine and further improvements}

\section{Proposed future approach of patient care pathway in patient- centred laboratory medicine}

Current approach in a clinic or small-scale hospital: Clinic examination of the patients is offered by medical doctors. The doctors give drugs to patients according to their symptoms and the physical examination. If the patient is not cured and revisits the clinic, the medical doctor may request a special clinical laboratory test for the patient. The medical laboratory will send a medical report to the corresponding doctor for final review and consideration. The medical doctor can adjust the treatment plan for the patient at the next visit. The problem of the current practice is that the time period taken to diagnose a disease is relatively long as it may rely on the experience of the doctors as well as self-alertness of the patient. For some severe cases like acute hepatitis, pathogenic infections or sepsis, the time for 


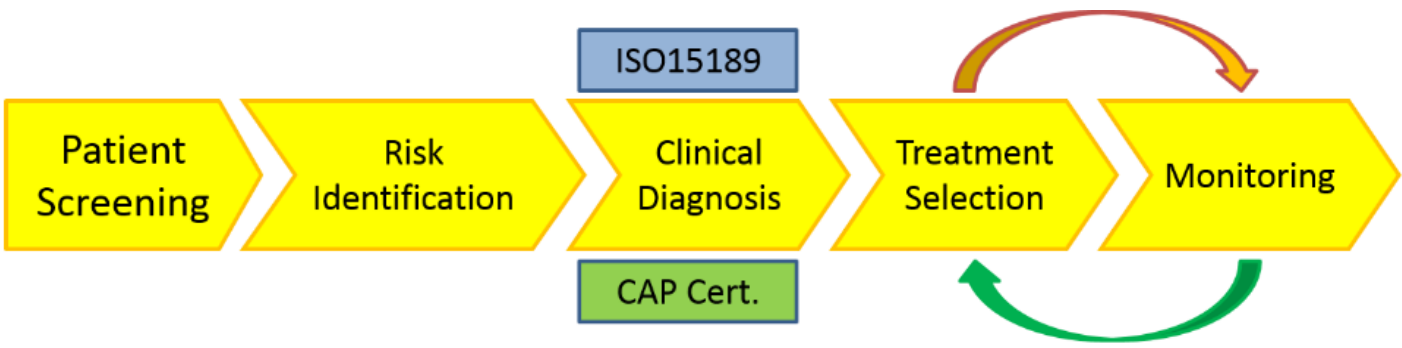

Figure 1. General medical laboratory processing. Five major parts in sequential order. patient screening, risk identification, clinical diagnosis, medical treatment selection and continuous monitoring. Treatment and monitoring will be adjusted from time to time according to the condition of the patients [7].

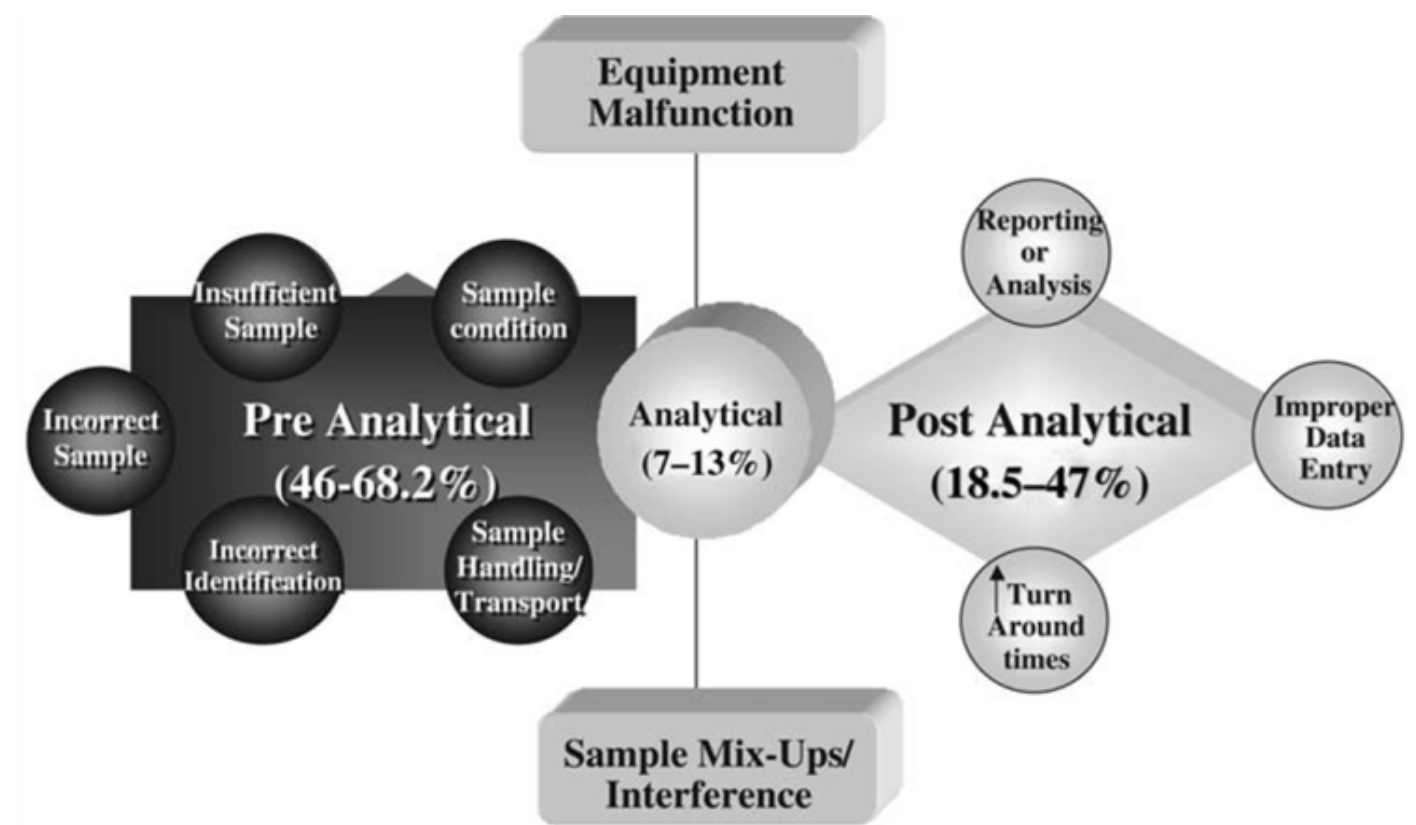

Figure 2. Common errors found in medical laboratory processing. Three types of errors could be identified. pre-analytical errors (sample collection and delivery), analytical errors (during testing) and post-analytical errors (data analysis and medical report preparation) [3].

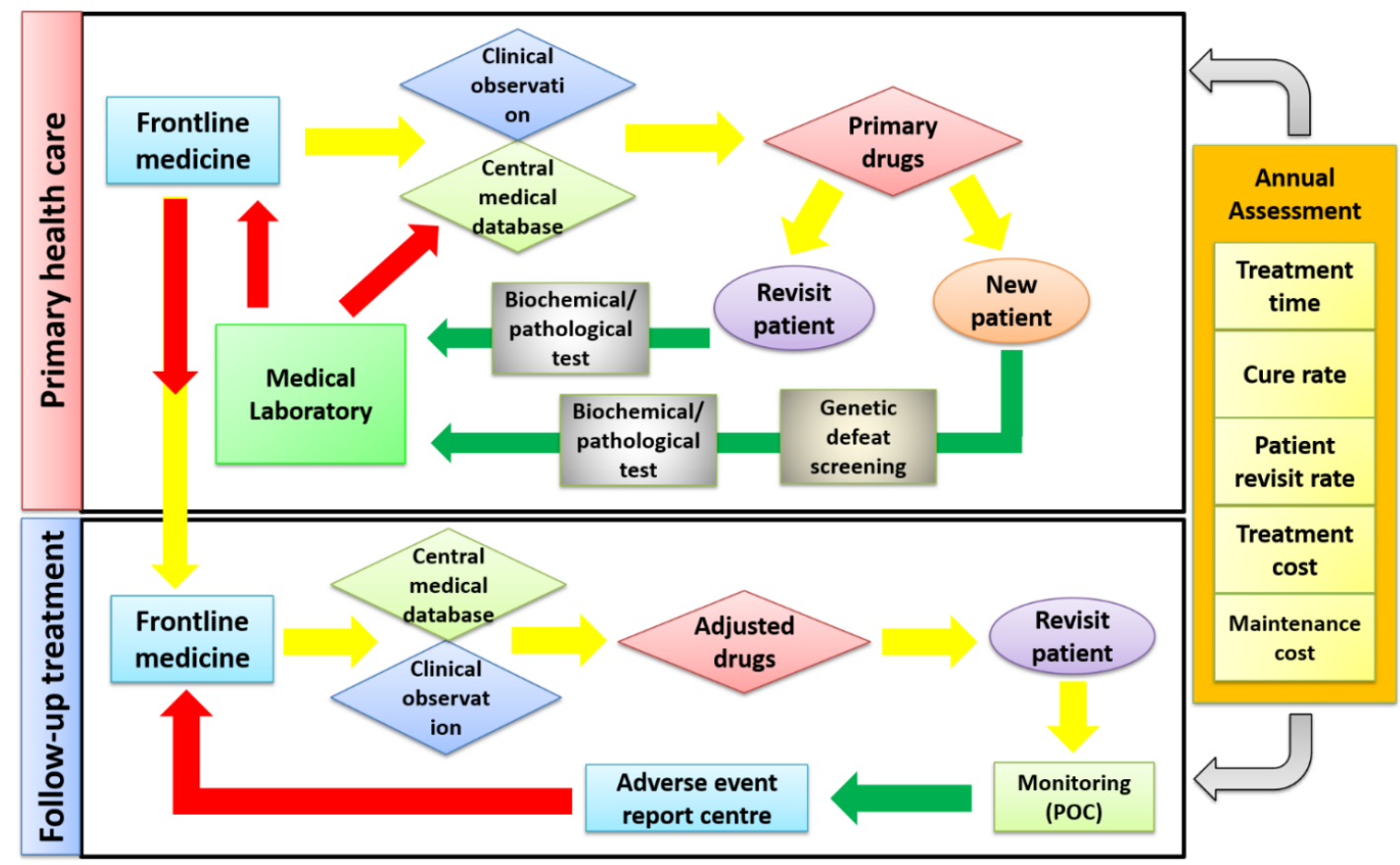

Figure 3. Suggested new approach of medical service in clinics and hospitals and the correspondent pathway. For consideration of the future medical service, suggestions are for at least four key stakeholders. patients, physicians and nurses in frontline medical unit, medical technologists for molecular diagnosis and health monitoring team. POC. point-of-care. 
treatment is extremely important. Rapid diagnosis with appropriate therapy for patients is pivotal. Hence, future patient-centred laboratory medicine should connect the frontline medical care (i.e. medical doctors and nurses) and clinical examination (i.e. medical diagnostic laboratory) as a general medical approach.

The medical record nowadays includes the treatment plans used, surgery record, medical examinations undertaken as well as some clinical results from the medical laboratory requested by the physicians. To augment the informativeness of the patient's record, consideration of the involvement of the genetic background may contribute to reduce the chances of developing drug allergy and other potential threat to the patient from the genetic defects. It is suggested that there could be an enhanced medical service for the public.

With reference to Dr. Price for the development of evidence-based laboratory medicine cycle $[10,11]$, a city with large populations could redevelop its clinical systems as follows: When a patient comes to the clinic, a medical doctor from frontline medicine (primary health care unit) should examine the symptoms of the patients and ask for the medical history. The doctor then may provide patients with primary drugs for treatment. Different patients are required to send their specimens (for example, blood, stool, urine or other body fluids) to the medical laboratory for molecular diagnosis. Some new patients may send their body cells for genetic defect screening while this procedure requires patient's consent. After that the medical laboratory will send a medical test report to the central medical database as well as a copy to frontline medicine department for backup. Doctors in the primary health care unit will give an appointment slip to the patient for followup treatment. A medical doctor from frontline medicine (follow-up treatment unit) then will adjust the drug combination and dosage in accordance with the medical test report, clinical observations as well as the genetic background of the patient. The patient will be given a basic POC device for regular health examination at home. Any adverse event will be sent to adverse event report centre followed by frontline medicine (follow-up treatment) for follow-up action (Figure 3). Based on the clinical diagnosis, and drug usage adjustment, this personalised therapeutic approach may be beneficial for improving the public health shield.

The management system should have regular assessments to maintain its quality. Frontline medicine-laboratory-monitoring system should have annual quality assessment under several quality management regulations like ISO or CAP systems. Main assessment criteria should be focused on the average treatment time for a patient, patient cure rate and revisit rate, treatment cost and maintenance cost. Some frontier laboratories like American Association for Clinical Chemistry have started to develop certain guidelines for quality management for laboratory medicine [12].

\section{Adaptation to new innovative applications and future laboratory medicine}

It is also suggested that a unit of research and development (R\&D) be established. This R\&D is independent of the testing department aims at designing and developing research studies for clinical application tests for new innovative biotechnology, assisting in technical transfer in clinical biochemistry, conducting certification and auditing for new clinical technologies $[7,13]$, improvement of current clinical laboratory examinations and assistance with internal auditor for quality management. It could be seen that the majority of devices and testing kits from clinical biotechnology companies have passed the quality control analysis while re-examination in the actual clinical laboratory is critical because of the environmental variations [14,15]. It is believed that new test analysis may maintain the precision and accuracy of the clinical tests in daily practice which could be functional in adjustment of testing procedures and develop troubleshooting guide for the staff (Figure 4).

Molecular diagnosis development is the future trend in medicine and has become the frontline in disease identification [16]. Genomics and biomarkers based personalised medicine in some human diseases like cancer therapy and cardiovascular diseases have been under clinical testing in recent years [17-19]. There is a systematic programme in computing based diagnostic analysis available for clinical science technologies which may facilitate the development of molecular diagnosis and new technology transfer [20].

\section{Change of work and responsibility of the laboratory staff: from "technician" to "technologist"}

In the past and even current practice in some district hospitals, the structure of a clinical laboratory usually follows the top-down approach of ordering and labour-dependent workflow, i.e. a medical doctor or a medical laboratory specialist like biochemical/biomedical professor with one to two technical officers (usually called chemists) managing the work of several senior members as medical technologists who supervise a large team of technicians. A medical doctor or medical laboratory specialist serves as the team head and consultant for final approval of the medical test report and quality management of the whole team. Technical officers are reviewers of the test results and they are responsible for managing the daily duties of the laboratory and maintaining the quality assurance management according to ISO15189. Medical laboratory technologist (MLT) as the team leader of the test work who is responsible for data analysis of test results, preparing patient's test report, guiding the routine work and providing technical support to technician. Technician is the person who runs the experiments assigned by MLT and assists the MLT in primary data analysis. However, by the innovation of automated systems and all-inone medical testing system, routine laboratory tests and primary data analysis are done by the automated testing-computing system (i.e. the system becomes "technician") and the actual technician shifts to be a MLT. Hence, job training for MLTs should be well planned especially for the freshly graduated employees. Although the technician workflow is relatively tedious, it may provide sufficient time to the new staff to practice and improve technical skills in laboratory works and further learn how to run for data analysis. After the induction automation, the trainee programme should be improved to adapt the integrated testing system.

For a typical training programme adapted to automated system for MLT, multiple key elements should be involved: basal and biochemical tests and diagnostic tests for half a year or above in order to reconstruct the laboratory practices as well as performance of an automated system of the new staff member who is certified and gets permission to work in an automated clinical laboratory. Examination for any performance assessment of the new staff shall be conducted by one to two senior MLTs or technical officers. It would be desirable if a joint-institutehospital programme is available for MTL students in the final year of their programme. After the practical assessment, the fresh MLT will conduct routine work in the laboratory for half a year to one year and then be admitted to the advanced training programme according to performance. The advanced training programme mainly focuses on the training of clinical reporting and technical support. The candidates should fully familiarize themselves with performing data analysis from 


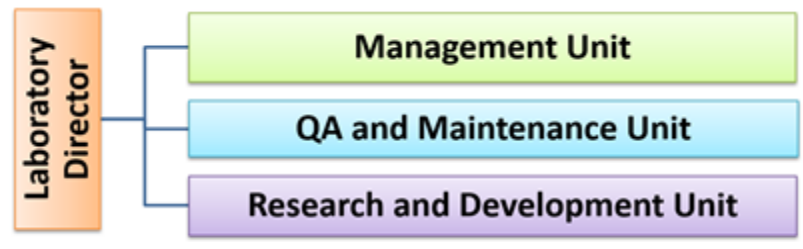

(A)
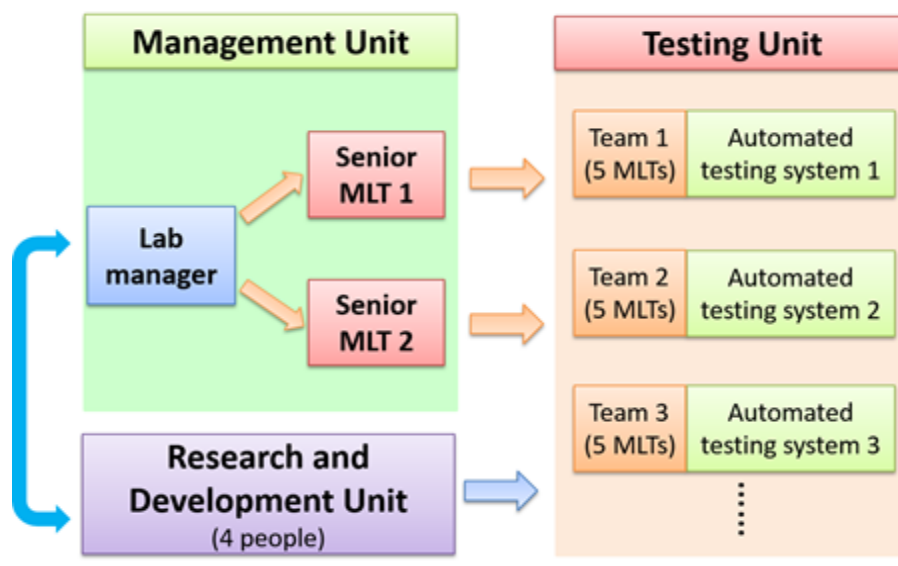

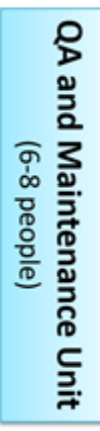

(B)

Figure 4. Proposed management scheme for an automated medical laboratory. A) Structure of laboratory management committee. The committee should include one laboratory director, one representative from management unit, research and development unit and, quality assurance (QA) and maintenance unit respectively. B) Infra-structure of medical laboratory. there are five key units in the laboratory system including management unit with laboratory manager and senior medical technologists (MLTs), testing unit with MLTs and automated systems, QA and maintenance unit and research and development unit.

the raw data provided and then making interpretation. They are required to write a certified clinical report to hospitals and medical doctors under the supervision of senior MTLs or chemists. They will also need to learn the procedures of troubleshooting regarding an automated system and the work of internal audit of the quality management system in the laboratory. Senior MLT will ensure laboratory quality control and assurance programming within the team, new staff training and technical improvement. Senior MLTs will be the final reviewers of the clinical test report. Technical officers will be responsible for managing the whole laboratory and serve as the internal auditor together with the laboratory head. They will also become the coordinators for technical transfer and technological innovation in laboratory medicine.

\section{Enhancement for management of medical laboratory}

Patient-centred medicine could be enhanced by advanced medical quality management as well as improvement of the structure of the medical laboratory. To maintain the quality system in a medical laboratory, a committee for quality assurance should be established to include a laboratory director, two representatives from management unit (MU) and frontline testing unit (TU), one from quality assurance and maintenance unit (QAMU) and one from research and development unit (RDU). The committee should hold an official meeting every season and an annual meeting with other medical departments to review the work in the medical laboratory and discuss the improvement and enhancement policies to the medical laboratory. Each unit, i.e. MU-TU, QAMU and RDU should report their work to the director monthly.

For the infra-structure of a medical laboratory, there are five key units in the laboratory system, namely, TU, MU, QAMU and RDU. $\mathrm{TU}$ is the largest and core unit in the laboratory consisting of several medical technologists (MLTs) and automated systems. Each team comprises 4 MLT II who prepare samples, monitor the automated system, record the test results and draft the medical report. One MLT I as a team leader is responsible for supporting MLT II, organizing daily duties of the team and reviewing the medical report. MU is the director and coordinator of TU which consists of one laboratory manager and two or more senior MLTs. Senior MLTs will be in charge of routine operation of the laboratory and the final reviewers of the medical report. They are responsible for report validation and arrangement of manpower. Laboratory Manager is the operative director of the MU and TU. He or she together with senior MLTs may regularly hold discussions with RDU for technical improvement. QAMU is quality guard in the medical laboratory. The QA team should pay visits to the laboratory regularly (like one examination per month and at least one internal audit per year) to check whether the laboratory operation, data processing and staff training fulfil the requirements of quality accreditation in accordance with ISO15189 or CAP accreditation. They participate in certification and amendment of standard operation protocols. The maintenance team is responsible for preparation of maintenance schemes, mechanical trouble shooting and electronic database management. QAMU is led by one QA manager with three to four members in QA branch and two to three members in maintenance branch. RDU is responsible for developing new technology researches in clinical sciences, technical transfer to TU and editing standard operation protocols with QA team. RDU usually has regular meetings with MU for arrangement of staff training. The RDU may have 4 members with expert experience in clinical sciences (Figure 4).

\section{Conclusion}

Laboratory medicine is the frontline medicine in disease diagnosis. due to the recent advanced development of molecular medicine and automation of the clinical system. It could be foreseen that laboratory medicine will contribute substantially to targeted therapy and personalised management, especially in cancers. However, systematic quality management should be rigorously maintained in order to provide high-quality service in medicine. 


\section{References}

1. Price CP (2003) Application of the principles of evidence-based medicine to laboratory medicine. Clinica Chimica Acta 332: 147-154

2. Price C, Christenson RH (2013) Ask the right question: a critical step for practicing evidence-based laboratory medicine. Ann Clin Biochem 50: 306-314. [Crossref]

3. Plebani M (2006) Errors in clinical laboratories or errors in laboratory medicine. Clin Chem Lab Med 44: 750-759. [Crossref]

4. International Organization for Standardization (2016) ISO 15189:2012(en) Medical Laboratories-requirements for Quality and Competence. [online] Available at: https:// www.iso.org/standard/56115.html [Accessed 6 June 2017].

5. Thelen MH, Vanstapel FJ, Kroupis C, Vukasovic I, Boursier G, et al. (2015) Flexible scope for ISO 15189 accreditation: a guidance prepared by the European Federation of Clinical Chemistry and Laboratory Medicine (EFLM) Working Group Accreditation and ISO/CEN standards (WG-A/ISO) Clin Chem Lab Med 53: 1173-1180. [Crossref]

6. College of American Pathologists (2017) Laboratory Accreditation Program. [Available at: http://www.cap.org/web/home/lab/accreditation?_adf.ctrl-state $=\mathrm{g} 34 \mathrm{rxqke} 0 \_4 \&$ afrLoop=219595060423806\#! ] [Accessed 6 June 2017].

7. Hallworth, MJ, Epner PL, Ebert C, Fantz, CR, Faye SA, et al. (2015) Current evidence and future perspectives on the effective practice of patient-centered laboratory medicine. Clin Chem 61: 589-599. [Crossref]

8. Horvath AR, Pewsner D (2004) Systematic reviews in laboratory medicine: principles, processes and practical considerations. Clin Chim Acta 342: 23-39. [Crossref]

9. Plebani M, Sciacovelli L, Aita A, Padoan A, Chiozza ML (2014) Quality indicators to detect pre-analytical errors in laboratory testing. Clin Chim Acta 432: 44-48. [Crossref]

10. Price CP (2012) Evidence-based laboratory medicine: is it working in practice. Clin Biochem Rev 33: 13-19. [Crossref]

11. Price CP (2000) Evidence-based laboratory medicine: supporting decision-making. Clin Chem 46: 1041-1050. [Crossref]
12. American Association for Clinical Chemistry (2015) Laboratory Medicine: Advancing Quality in Patient Care, AACC Policy Reports. [Available at: https://www.aacc.org/ health-and-science-policy/aacc-policy-reports/2015/laboratory-medicine-advancingquality-in-patient-care] [Accessed 7 June 2017].

13. Beastall GH (2013) Adding value to laboratory medicine: a professional responsibility. Clin Chem Lab Med 51: 221-227. [Crossref]

14. Philips Z, Ginnelly L, Sculpher M, Claxton K, Golder Set, et al. (2004) Review of guidelines for good practice in decision-analytic modelling in health technology assessment. Health Technol Assess 8: 1-158. [Crossref]

15. Philips Z, Bojke L, Sculpher M, Claxton K, Golder S (2006) Good practice guidelines for decision-analytic modelling in health technology assessment: a review and consolidation of quality assessment. Pharmacoeconomics 24: 355-371. [Crossref]

16. Pirmohamed M (2010) Acceptance of biomarker-based tests for application in clinical practice: criteria and obstacles. Clin Pharmacol Ther 88: 862-866. [Crossref]

17. Ginsburg GS, Kuderer NM (2012) Comparative effectiveness research, genomicsenabled personalized medicine, and rapid learning health care: a common bond. J Clin Oncol 30: 4233-4242. [Crossref]

18. Kelley RK, Van Bebber, SL, Phillips KA, Venook AP (2011) Personalized medicine and oncology practice guidelines: a case study of contemporary biomarkers in colorectal cancer. J Natl Compr Canc Netw 9: 13-25. [Crossref]

19. Völzke H, Schmidt CO, Baumeister SE, Ittermann T, Fung G, et al. 2(013) Personalized cardiovascular medicine: concepts and methodological considerations. Nat Rev Cardiol 10: 308-316. [Crossref]

20. Main C, Moxham T, Wyatt JC, Kay J, Anderson R (2010) Computerised decision support systems in order communication for diagnostic, screening or monitoring tes ordering: systematic reviews of the effects and cost-effectiveness of systems. Health Technol Assess 14: 1-227. [Crossref]

Copyright: (C2017 Tam C. This is an open-access article distributed under the terms of the Creative Commons Attribution License, which permits unrestricted use, distribution, and reproduction in any medium, provided the original author and source are credited. 